\title{
Mitochondrial Epigenetic Changes Link to Increased Diabetes Risk and Early-Stage Prediabetes Indicator
}

\author{
Louise D. Zheng, ${ }^{1}$ Leah E. Linarelli, ${ }^{1}$ Joseph Brooke, ${ }^{1}$ Cayleen Smith, \\ Sarah S. Wall, ${ }^{1}$ Mark H. Greenawald, ${ }^{2}$ Richard W. Seidel, ${ }^{3}$ Paul A. Estabrooks, ${ }^{1,2,4}$ \\ Fabio A. Almeida, ${ }^{1,4}$ and Zhiyong Cheng ${ }^{1}$
}

\author{
${ }^{1}$ Department of Human Nutrition, Foods, and Exercise, Fralin Translational Obesity Research Center, \\ College of Agriculture and Life Science, Virginia Tech, Blacksburg, VA 24061, USA \\ ${ }^{2}$ Department of Family and Community Medicine, Carilion Clinic, Roanoke, VA 24016, USA \\ ${ }^{3}$ Department of Psychiatry, Carilion Clinic, Roanoke, VA 24014, USA \\ ${ }^{4}$ Department of Health Promotion, Social \& Behavioral Health, College of Public Health, University of Nebraska Medical Center, \\ Omaha, NE 68198, USA
}

Correspondence should be addressed to Fabio A. Almeida; fabio.almeida@unmc.edu and Zhiyong Cheng; zcheng@vt.edu

Received 13 January 2016; Accepted 26 April 2016

Academic Editor: Saeid Golbidi

Copyright (C) 2016 Louise D. Zheng et al. This is an open access article distributed under the Creative Commons Attribution License, which permits unrestricted use, distribution, and reproduction in any medium, provided the original work is properly cited.

\begin{abstract}
Type 2 diabetes (T2D) is characterized by mitochondrial derangement and oxidative stress. With no known cure for T2D, it is critical to identify mitochondrial biomarkers for early diagnosis of prediabetes and disease prevention. Here we examined 87 participants on the diagnosis power of fasting glucose (FG) and hemoglobin Alc levels and investigated their interactions with mitochondrial DNA methylation. FG and Alc led to discordant diagnostic results irrespective of increased body mass index (BMI), underscoring the need of new biomarkers for prediabetes diagnosis. Mitochondrial DNA methylation levels were not correlated with late-stage (impaired FG or Alc) but significantly with early-stage (impaired insulin sensitivity) events. Quartiles of BMI suggested that mitochondrial DNA methylation increased drastically from Q1 $(20<\mathrm{BMI}<24.9$, lean) to Q2 $(30<\mathrm{BMI}<34.9$, obese), but marginally from Q2 to Q3 (35< BMI < 39.9, severely obese) and from Q3 to Q4 (BMI > 40, morbidly obese). A significant change was also observed from Q1 to Q2 in HOMA insulin sensitivity but not in A1c or FG. Thus, mitochondrial epigenetic changes link to increased diabetes risk and the indicator of early-stage prediabetes. Further larger-scale studies to examine the potential of mitochondrial epigenetic marker in prediabetes diagnosis will be of critical importance for T2D prevention.
\end{abstract}

\section{Introduction}

Lifestyle contributes significantly to the epidemic of type 2 diabetes (T2D) [1-4]. The interactions between lifestyle factors and mitochondria (the primary metabolic platform) have been implicated in the pathogenesis of diabetes and obesity [5]. In particular, mitochondria undergo aberrant alteration and cause oxidative stress in diabetic subjects, and redox active compounds have shown promising potential to treat T2D [5-8]. Nevertheless, no known cure has been developed for diabetes to date $[9,10]$, and early diagnosis of prediabetes for intervention has been an important strategy to prevent T2D [3]. To this end, biomarkers that can signify progression or early stage of prediabetes will be of clinical significance.

The risk of T2D increases with weight gain. For instance, the lifetime risk of developing diabetes at an age of 18 years increase by $9.9 \%, 37.2 \%$, and $50.5 \%$ in overweight $(25<\mathrm{BMI}$ $<30)$, obese $(30<\mathrm{BMI}<35)$, and severely obese $(>35)$ males, respectively, compared with normal weight individuals; for female subjects, it increases by $18.3 \%, 37.5 \%$, and $57.3 \%$, respectively [11]. As such, the American Diabetes Association (ADA) recommends BMI as a primary index to screen for high-risk population and recommend of further testing to 
assess risk of future diabetes development in asymptomatic people [12]. Following an initial weight screening, individuals having a fasting glucose (FG) ranging from $100 \mathrm{mg} / \mathrm{dL}$ $(5.6 \mathrm{mmol} / \mathrm{L})$ to $125 \mathrm{mg} / \mathrm{dL}(6.9 \mathrm{mmol} / \mathrm{L})$ or an Alc of $5.7-$ $6.4 \%$ are diagnosed as prediabetic [12]. Of note, the existing discordance between FG and Alc may lead to conflicting results, underscoring the utmost importance of identifying new markers that can consistently identify prediabetic individuals as early as possible for T2D prevention [13-15].

The effectiveness of diabetes prevention via lifestyle modification is strongly associated with improved insulin sensitivity [16] and epigenetic adaptation, including DNA methylation on the nuclear genome and mitochondrial genome [5, 17]. Recently, we identified an insulin signalingepigenetic-genetic axis in the regulation of mitochondria, the major cellular metabolic platform that responds robustly to interventions $[6,18-23]$. However, it is largely unknown whether mitochondrial epigenetic signature reflects prediabetes progression and how it interacts with the current diagnostic indices (Alc and FG). In the present work, we studied the interactions of mitochondrial DNA methylation with FG, Alc, and insulin sensitivity, the parameters that change stage-dependently during prediabetes progression [24-26]. DNA methylation in mitochondrial NADH dehydrogenase 6 (ND6) and displacement loop (D-loop) region was significantly correlated with changes in insulin sensitivity (the event starting in an earlier stage [24-26]) but had marginal interaction with Alc and FG (the later-stage markers in prediabetes progression [24-26]). Upon quartilizing BMI (the primary risk index for prediabetics screening [11, 12]), we found significant changes in mitochondrial epigenetics during the progression from Q1 $(20<\mathrm{BMI}<24.9$, lean $)$ to Q2 $(30<\mathrm{BMI}<34.9$, obese), but not from Q2 to Q3 $35<$ $\mathrm{BMI}<39.9$, severely obese) or Q3 to Q4 (BMI > 40, morbidly obese); by contrast, FG showed significant changes only from Q3 to Q4, and the levels of A1c were unchanged in all cases. Discordance was observed when FG or Alc was used to diagnose prediabetes, presumably because they indicate late but different stages of prediabetes. Together, our data suggest that mitochondrial epigenetic changes link to diabetes risk and signify early-stage prediabetes, targeting which may have the potential to achieve early diagnosis of prediabetes for T2D intervention.

\section{Materials and Methods}

2.1. Ethics Statement. A written informed consent was obtained from each participant. All procedures were conducted in accordance with NIH Guidelines, and the research protocol was approved by Institutional Review Boards (IRB) at Carillion Clinic and at Virginia Tech.

2.2. Study Patients, Sample Inclusion, and Blood Chemistry. This study included 87 participants at baseline (before intervention), which were recruited for a larger diabetes prevention trial (diaBEAT-it trial) [27]. Twelve individuals were lean, and 75 participants were obese or overweight (Table 1). All participants completed an intake questionnaire
TABLE 1: Demographic and metabolic characteristics of participants.

\begin{tabular}{lcc}
\hline Characteristics & $\begin{array}{c}\text { Lean } \\
(n=12)\end{array}$ & $\begin{array}{c}\text { Overweight/obese } \\
(n=75)\end{array}$ \\
\hline Sex (male/female) & $3 / 9$ & $22 / 53$ \\
Age (years) & $25.7 \pm 3.1$ & $48.4 \pm 1.5^{* * *}$ \\
BMI (kg/m $\left.{ }^{2}\right)$ & $22.7 \pm 0.6$ & $37.1 \pm 0.8^{* * *}$ \\
Fasting glucose (mg/dL) & $83.4 \pm 1.8$ & $96.1 \pm 1.5^{* *}$ \\
Fasting insulin $(\mu \mathrm{IU} / \mathrm{mL})$ & $10.0 \pm 1.5$ & $24.6 \pm 2.4^{*}$ \\
HOMA-IR & $2.1 \pm 0.3$ & $6.0 \pm 0.6^{*}$ \\
HbAlc $(\%)$ & $5.5 \pm 0.1$ & $5.7 \pm 0.1$ \\
HDL (mg/dL) & $61.9 \pm 4.2$ & $54.7 \pm 1.4$ \\
LDL (mg/dL) & $90.8 \pm 6.7$ & $114.1 \pm 3.5^{*}$ \\
LDL/HDL ratio & $1.6 \pm 0.2$ & $2.2 \pm 0.1^{*}$ \\
VLDL (mg/dL) & $17.6 \pm 2.2$ & $28.4 \pm 2.0^{*}$ \\
Total cholesterol (mg/dL) & $170.1 \pm 5.5$ & $195.9 \pm 3.9^{*}$ \\
Total cholesterol/HDL & $2.9 \pm 0.2$ & $3.7 \pm 0.1^{* *}$ \\
Triglyceride (mg/dL) & $88.1 \pm 10.9$ & $140.7 \pm 10.1^{*}$ \\
\hline
\end{tabular}

Mean \pm SE; ${ }^{*} p<0.05 ;{ }^{* *} p<0.01 ;{ }^{* * *} p<0.001$.

about medical history, current medications, and current health behaviors. For blood collection, the participants were instructed to fast overnight (10-12 hours) before their scheduled blood draw at Solstas Labs facility (Roanoke, Virginia) [18]. Fasting venous blood samples were collected in EDTA tubes to study lipid profile (triglyceride, total cholesterol, HDL-cholesterol, and LDL-cholesterol), fasting plasma glucose, fasting plasma insulin, and HbAlc. The homeostasis model assessment for insulin resistance (HOMA-IR) index was calculated by (fasting insulin $(\mu \mathrm{IU} / \mathrm{mL}) \times$ fasting glucose $(\mathrm{mg} / \mathrm{dL}) / 405)$ as previously described $[18,28,29]$. In addition, buffy coats (white blood cells) were prepared from fasting blood samples and stored at $-80^{\circ} \mathrm{C}$ for later DNA extraction and methylation analysis $[18,30,31]$.

2.3. DNA Isolation and Bisulfite Conversion. Fast extraction of high-quality DNA from buffy coats was conducted with QIAamp DNA Blood Mini Kits (Qiagen, Hilden, Germany), which utilize a silica-membrane-based DNA purification. DNA quality and quantity were determined on a Synergy H4 Hybrid Multi-Mode Microplate Reader (BioTek Instruments, Winooski, VT, USA). For bisulfite treatment and purification of converted DNA, we used EpiTect Bisulfite Kits (Qiagen). During bisulfite treatment, the methylated cytosines were conserved but unmethylated cytosines converted into uracils, and the purified DNA was used for methylation analysis [18].

2.4. Mitochondrial DNA Methylation. Methylation specific PCR (MSP) was employed to analyze mitochondrial DNA methylation in ND6 and D-loop region [18, 32, 33]. The bisulfite modified DNA was used as a template for MSP reactions on a ViiA ${ }^{\mathrm{TM}} 7$ Real-Time PCR System, using $\mathrm{iQ}^{\mathrm{TM}} \mathrm{SYBR}^{\circledR}$ Green Supermix (Bio-Rad Laboratories, Hercules, CA, USA). Two MSPs were performed simultaneously to detect the methylated and unmethylated DNA for each sample, and the 
percentage of methylated DNA was calculated as described previously $[18,32,33]$. The primers used in this study were TAGGAATTAAAGATAGATATTGCGA (forward) and $5^{\prime}$-ACTCTCCA TACATTTAATATTTTCGTC- ${ }^{\prime}$ (reverse) for methylated D-loop; 5'-GGTAGGAATTAAA GATAGATATTGTGA-3' (forward) and $5^{\prime}$-ACTCTCCATACATTTAATATTTTCATC- $3^{\prime}$ (reverse) for unmethylated D-loop; $5^{\prime}$-TTTCGTATTAATAGGATTTTTTCGA-3' (forward) and $5^{\prime}$-AATTATCTTTAAATATACTACAACGAT-3' (reverse) for methylated ND6; $5^{\prime}$-TTTTGTATTAATAGGATTTTTTTGA-3' (forward) and $5^{\prime}$-ATAATTATCTTTAAATAT ACTACAACAAT- ${ }^{\prime}$ (reverse) for unmethylated ND6 [32]. Primer design was performed with the MethPrimer program, based on the sequence of human mitochondrial genome (>gi|251831106|ref|NC_012920.1|Homo sapiens mitochondrion, complete genome) [34].

2.5. Statistical Analysis. Pearson's correlation and regression analysis were applied to examine the relationships among obesity-related risk factor, metabolic indexes, and mitochondrial DNA methylation levels. In some cases, logarithmtransformed data were used for skewed variables (e.g., HOMA-IR). The data were expressed as the mean \pm SE unless otherwise specified, and analysis of variance (ANOVA) was conducted to determine $p$ values; $p<0.05$ was considered statistically significant.

\section{Results}

3.1. Metabolic Changes with BMI. Compared with the lean $(\mathrm{BMI}=22.7 \pm 0.6)$ group, overweight and obese $(\mathrm{BMI}=$ $37.1 \pm 1.8$ ) individuals showed significant elevation in fasting glucose (83.4 versus $96.1 \mathrm{mg} / \mathrm{dL}, p<0.01)$ and insulin $(10.0$ versus $24.6 \mu \mathrm{IU}, p<0.05$ ) levels (Table 1 ). In line with the notion that an increase in fasting insulin (FI) indicates impairment of insulin signaling $[35,36]$, we found that the homeostasis model assessment-estimated insulin resistance (HOMA-IR) index increased from 2.1 in the lean group to 6.0 in the overweight and obese group $(p<0.05)$ (Table 1$)$. In addition, lipid profile was dysregulated in the overweight and obese individuals, showing significant elevation in LDL, VLDL, total cholesterol, and triglyceride (Table 1). However, Alc showed no difference between the two groups (5.5 versus 5.7, $p>0.05)$. These data suggest that BMI can reflect the changes in glucose and lipid metabolism, as well as the impairment in insulin signaling, supporting the notion that BMI represents an important risk index for both prediabetes and T2D development [11, 37, 38].

3.2. Diagnosis of Prediabetes Using A1c and FG. The observation of no difference in Alc between the low-risk (BMI = $22.7 \pm 0.6)$ and high-risk (BMI $=37.1 \pm 1.8$ ) groups prompted us to examine the power of Alc in diagnosing prediabetes. According to the standards recommended by ADA [12], Alc indicated that $49.4 \%$ of the 87 participants had prediabetes $(\mathrm{Alc}=5.7-6.4 \%), 3.5 \%$ had $\mathrm{T} 2 \mathrm{D}(\mathrm{Alc} \geq$ $6.5 \%)$, and $47.1 \%$ were considered healthy (Alc $=4.8-5.6 \%)$. However, FG suggested that $71.3 \%$ of the participants were healthy, $27.6 \%$ were prediabetic, and only $1.1 \%$ had T2D (Figure 1). This discordance in Alc and FG recapitulates the findings in previous reports and underscores the urgent need of additional markers for reliable and consistent diagnosis [13-15].

3.3. Insulin Resistance Reflected Diabetes Risk Better than $A 1 c$ and FG. Prediabetes is characterized by impaired fasting glucose and insulin sensitivity, which progress differently from Alc before the onset of T2D (Supplemental Figure 1 in Supplementary Material available online at http://dx.doi.org/10.1155/2016/5290638) [24-26]. Specifically, drastic changes were observed in insulin sensitivity 5 years prior to the diagnosis of T2D, which is 3 years earlier than FG and 4 years earlier than A1c (Supplemental Figure 1) [24$26]$. In the lean or healthy group $(\mathrm{BMI}=22.7 \pm 0.6)$, regression analysis showed no significant correlation of BMI with FG $(p=0.5876)$, Alc $(p=0.1052)$, FI $(p=0.4095)$, or HOMAIR $(p=0.4752)$ (Figure 2). Surprisingly, FG and Alc also failed to correlate with BMI in the high-risk $(B M I=37.1 \pm$ 1.8 ) group ( $p=0.3677$ and 0.4865 , resp., Figures $2(a)$ and 2(b)). By contrast, FI and HOMA-IR showed significant correlation with BMI ( $p=0.0056$ and 0.0086 , resp., Figures 2(c) and 2(d)). Logarithm-transformed HOMA-IR values showed a similar correlation with BMI (Supplemental Figure 2). These data suggest that insulin resistance indices can better reflect diabetes risk. Given that impaired insulin sensitivity is observed prior to impaired FG and Alc in prediabetes [2426], use of insulin resistance index may be superior to Alc or FG for early detection of prediabetes.

3.4. Mitochondrial Epigenetic Changes Were Associated with Insulin Resistance. The onset of T2D in prediabetic individuals can be effectively delayed or prevented by lifestyle intervention, in response to which mitochondria undergo epigenetic adaptation [1-3, 5, 17, 18, 39]. To examine how mitochondrial epigenetics interacts with Alc, FG, and insulin resistance, we collected additional blood samples from a subset (40 out of the 87 , including 8 lean/healthy, and 32 obese) of participants and analyzed DNA methylation in mitochondrial ND6 and D-loop, the regions critical for mitochondrial function and DNA replication [18, 32]. The 40 individuals represented well the 87 participants, as demonstrated by the relations of BMI with Alc, FG, FI, and HOMA-IR (Supplemental Figure 3). More importantly, ND6 methylation level showed significant correlation with insulin resistance indices ( $p=0.0054$ for HOMA-IR and 0.0110 for FI; Figures 3(a) and 3(b)), but it had marginal interaction with FG $(p=0.1581$; Figure 3(c) ) and Alc $(p=0.3538$; Figure 3(d)). Further regression analyses suggested that ND6 methylation had no significant correlation with lipid profile or age (Supplemental Figure 4, A-D, or data not shown), consistent with previous studies of global DNA methylation $[40,41]$. This phenotype was recapitulated by D-loop methylation, which was significantly correlated with insulin resistance (HOMA-IR, $p=0.0337$; Figure $3(\mathrm{e})$ ), but not with Alc $(p=0.7427$; Figure 3(f) $)$ or FG $(p=0.7713$; Figure $3(\mathrm{~g}))$ or lipid profile (Figure 3(h), Supplemental Figure 4, E-H). 


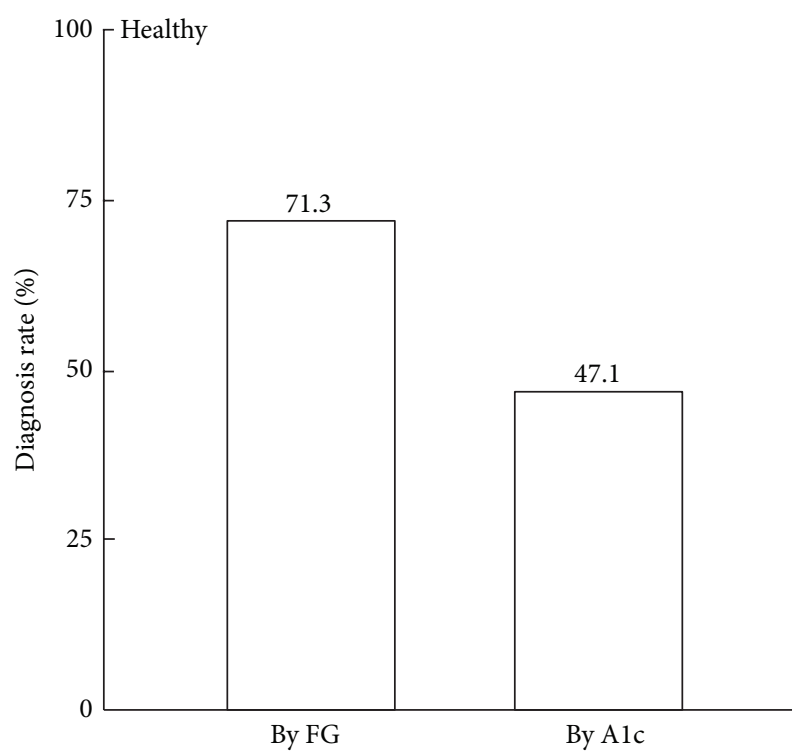

(a)

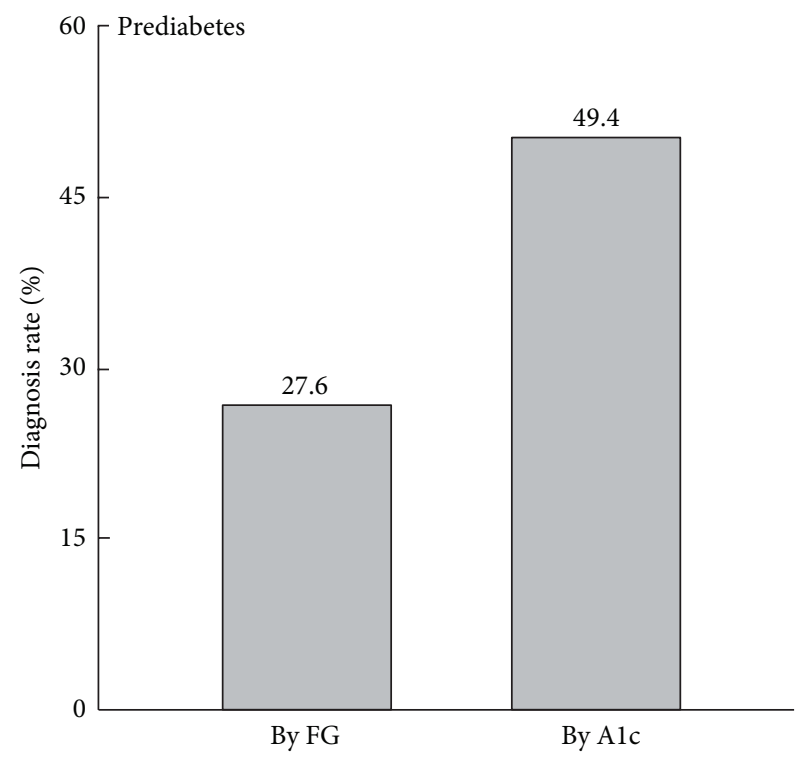

(b)

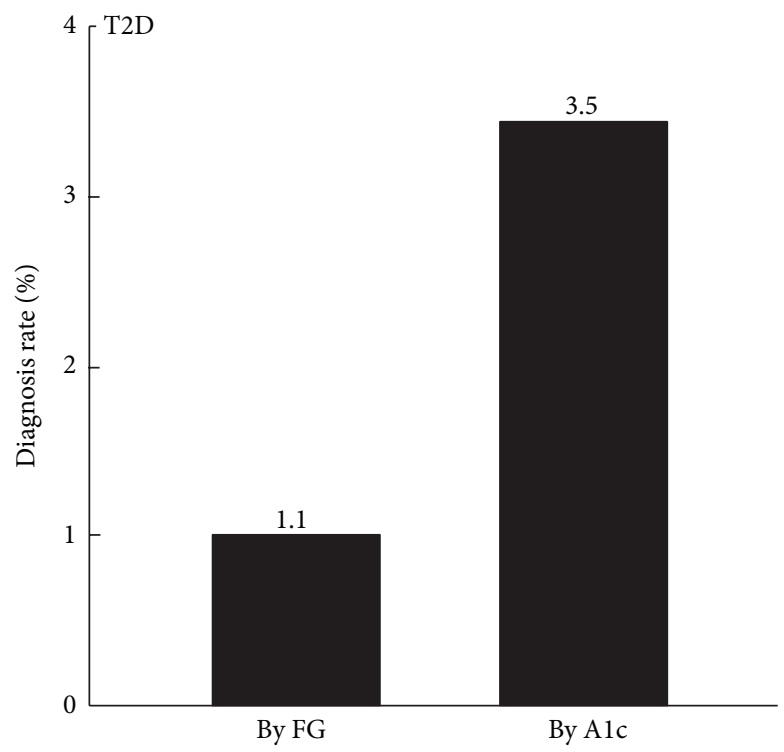

(c)

FIGURE 1: The diagnosis of healthy (a), prediabetes (b), and T2D (c) among 87 participants using the standards recommended by ADA for fasting glucose (FG) and hemoglobin Alc (Alc).

These data suggest that mitochondrial epigenetic signature may represent an early-stage marker of prediabetes and its alteration parallels with the development of insulin resistance.

3.5. Mitochondrial Epigenetic Traits Were Associated with Increase Diabetes Risk and Signified Early-Stage Prediabetes. Weight gain is an established risk factor for prediabetes and T2D: an increase in BMI indicates a higher risk [11, 37, 38]. Intriguingly, DNA methylation levels of both ND6 and D-loop showed significant correlation with BMI ( $p=$ 0.0295 and 0.0252 , resp., Figures $4(\mathrm{a})$ and $4(\mathrm{~b}))$. To test how mitochondrial epigenetic changes progress with this risk factor, we plotted the epigenetics data with quartiles of BMI: 20-24.9 (Q1, lean or healthy), 30-34.9 (Q2, obese), 3539.9 (Q3, severely obese), and >40 (Q4, morbidly obese). In comparison with Q1, the other three quartiles all showed significant increase in ND6 and D-loop methylation (Figures 4(c) and 4(d)). However, no further significant changes in DNA methylation were observed from Q2 to Q3 or to Q4. By contrast, insulin resistance indices (HOMA-IR and FI) underwent significant elevation from Q1 to Q2 and additionally from Q3 to Q4 (Figures 4(e) and 4(f)). The continuous changes in insulin resistance indices during high-risk stages (Q3 and Q4) are consistent with the trajectory showing that HOMA insulin sensitivity decreased steeply until 


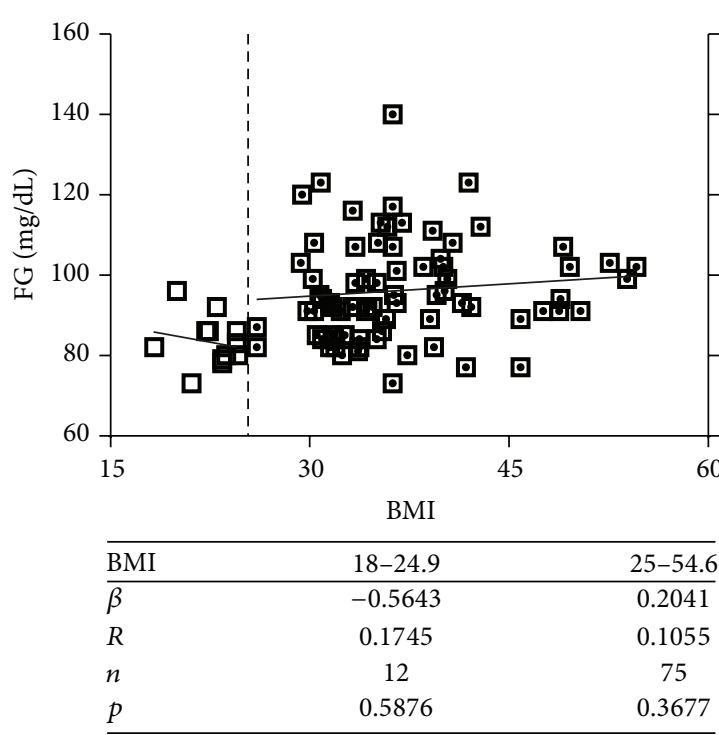

(a)

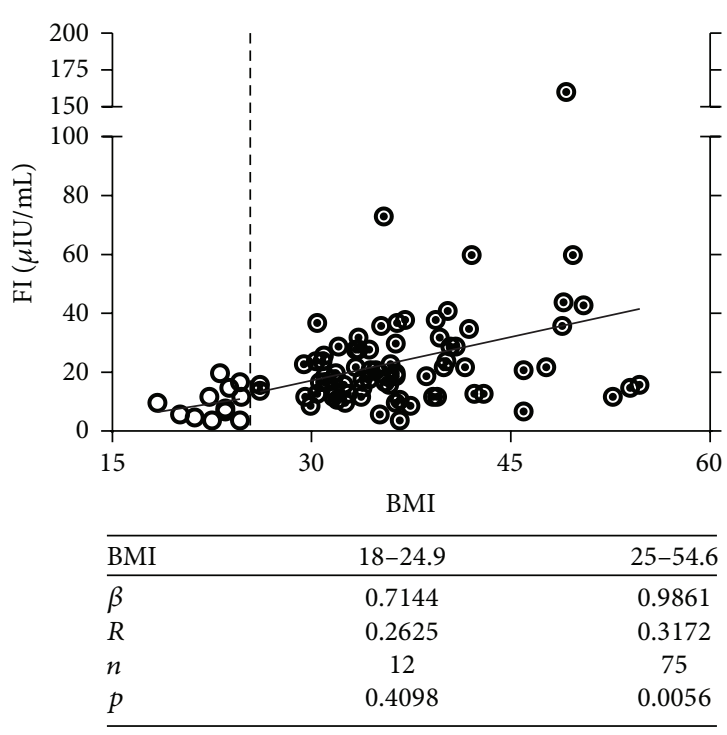

(c)

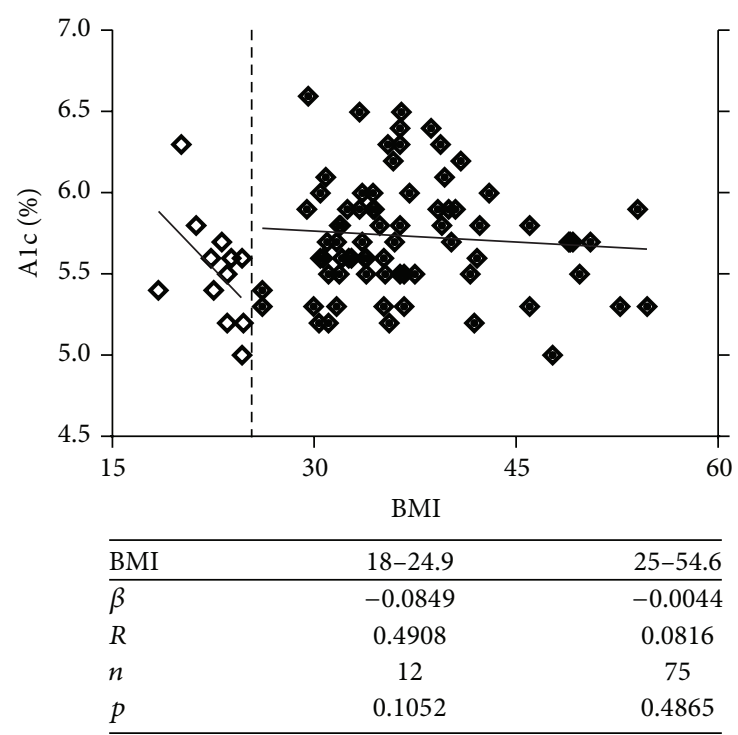

(b)

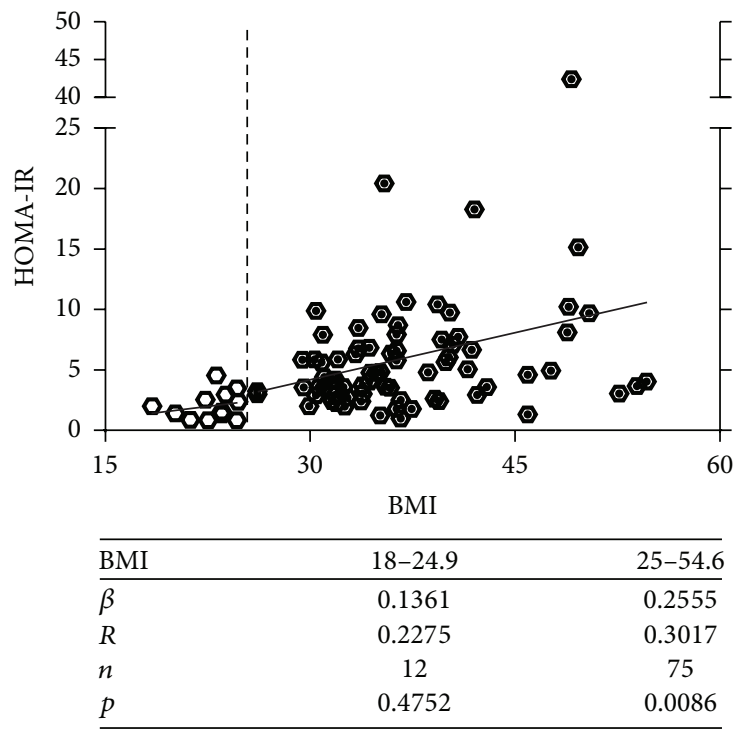

(d)

FIGURE 2: The correlations of obesity-associated diabetes risk index (BMI) with current diagnostic indices and insulin sensitivity. (a) FG showed no significant correlation with BMI. (b) Alc showed no significant correlation with BMI. (c-d) Decrease of insulin sensitivity was significantly associated with BMI in overweight and obese participants, as indicated by FI (c) and HOMA-IR (d).

the diagnosis of T2D (Supplemental Figure 1) [24, 25]. Of note, Alc showed marginal changes in all stages (from Q1 to Q4), and a significant increase in FG was observed only during advanced progression (Q3 and Q4) stages (Supplemental Figure 5). These results support the notion that FG is a late-stage marker of prediabetes progression, and change of Alc is an even later-stage event (Supplemental Figure 1) [2426]. Importantly, we found that mitochondrial epigenetic changes were most sensitive when prediabetes progressed from low-risk (Q1) to high-risk (Q2) stages but less responsive to further progression in higher risk (Q3 and Q4) stages. Therefore, targeting mitochondrial epigenetic markers has the potential to identify early-stage prediabetes for as-earlyas-possible lifestyle intervention.

\section{Discussion}

DNA methylation is a reversible epigenetic process that regulates genetic predisposition and gene expression and interacts with environmental cues [5, 40,41]. The global DNA methylation and its interaction with age, metabolic markers, sex hormone, inflammation, and lifestyle behaviors in T2D and obese patients have been investigated in previous studies [40-45]. Earlier studies suggested that global DNA 


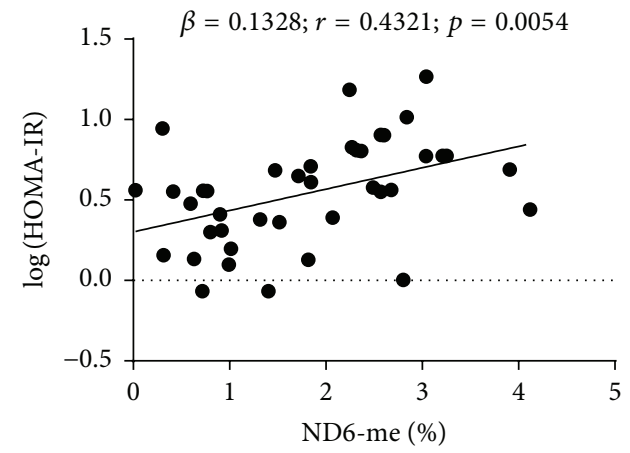

(a)

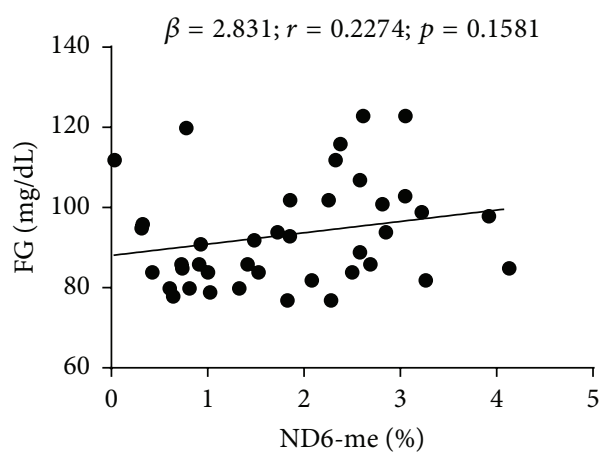

(c)

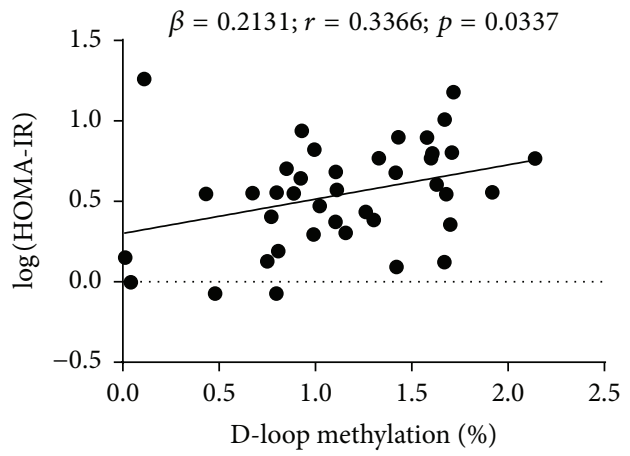

(e)

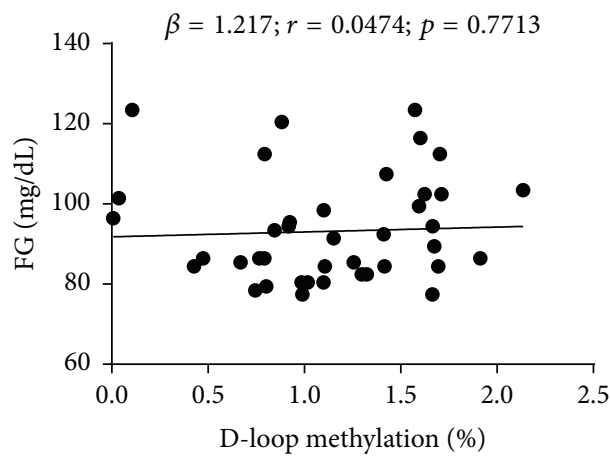

(g)

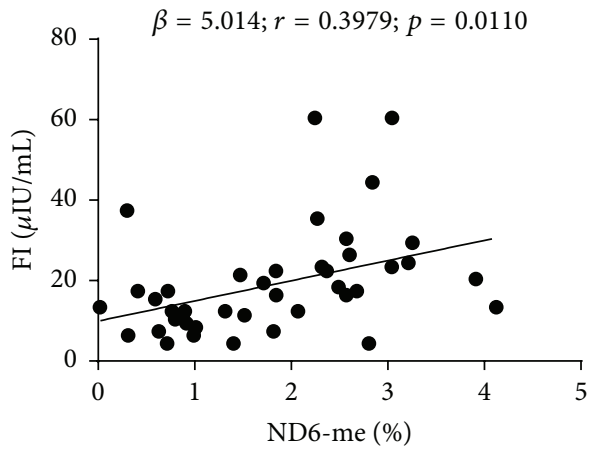

(b)

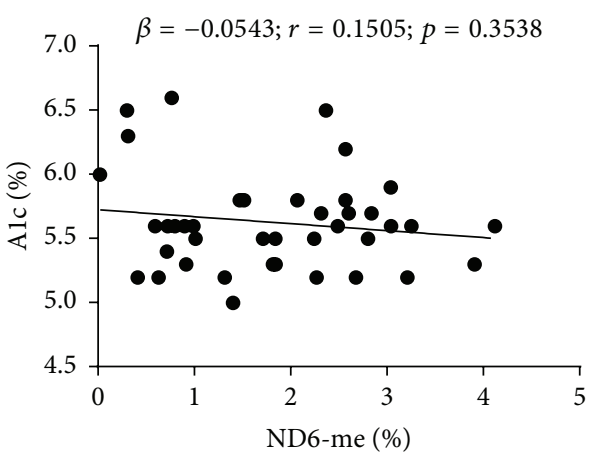

(d)

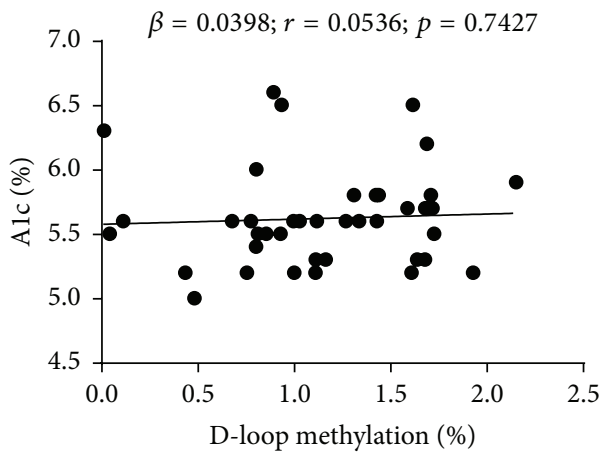

(f)

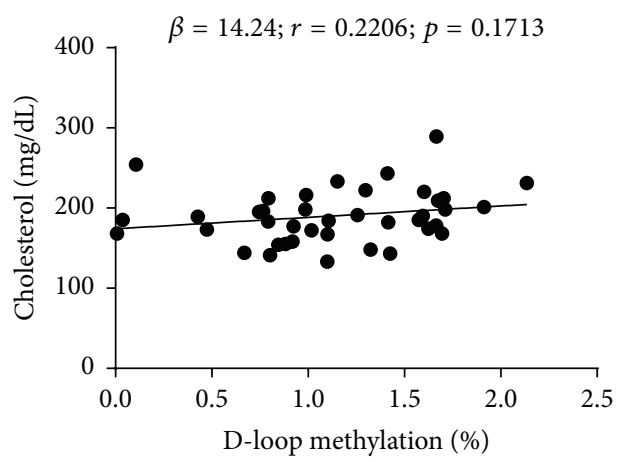

(h)

FIGURE 3: Correlation of mitochondrial DNA methylation with insulin sensitivity (HOMA-IR and FI) and current diagnostic indices (FG and Alc). (a-b) HOMA-IR and FI showed significant interaction with ND6 methylation; $n=40$. (c) FG showed no correlation with ND6 methylation; $n=40$. (d) Alc showed no correlation with ND6 methylation; $n=40$. (e) HOMA-IR showed significant interaction with D-loop methylation; $n=40$. (f) FG showed no correlation with D-loop methylation; $n=40$. (g) Alc showed no correlation with D-loop methylation; $n=40$. (h) Cholesterol showed no correlation with D-loop methylation; $n=40$. More details about correlation of DNA methylation in mitochondrial D-loop with lipid profile can be found in Supplemental Figure 4. 


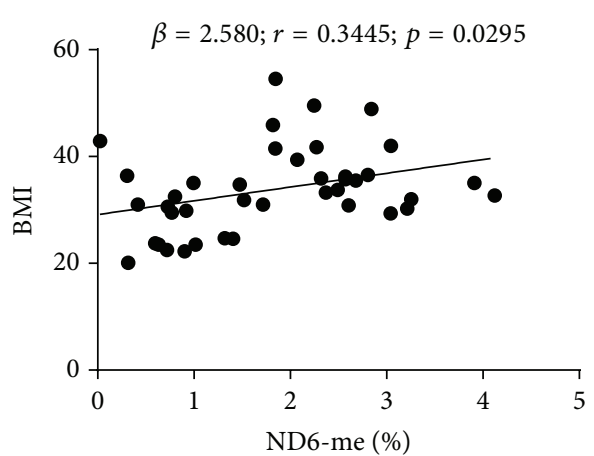

(a)

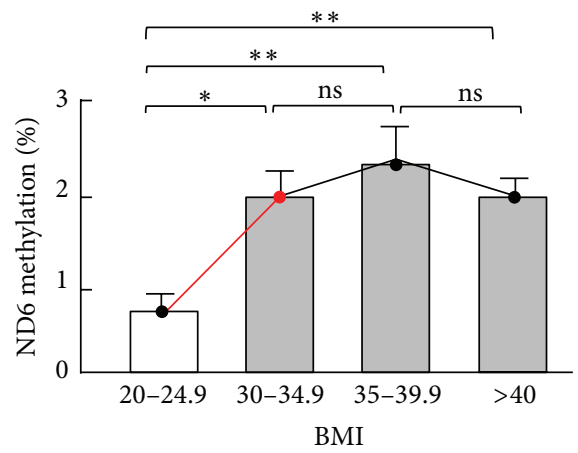

(c)

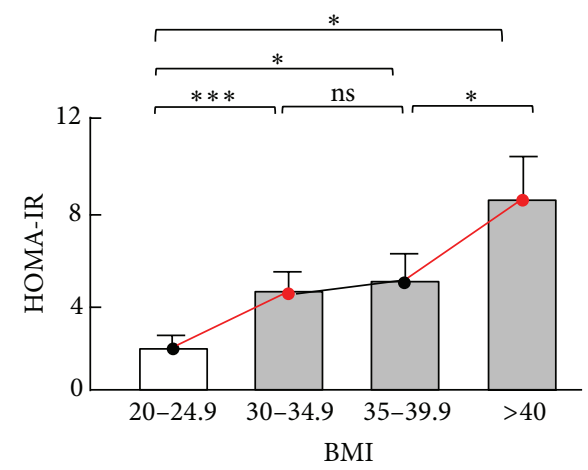

(e)

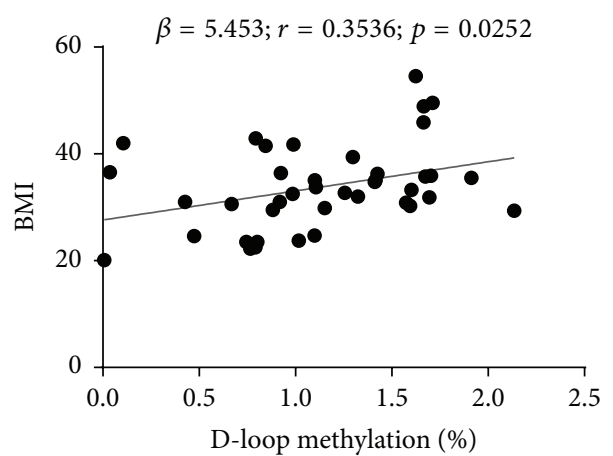

(b)

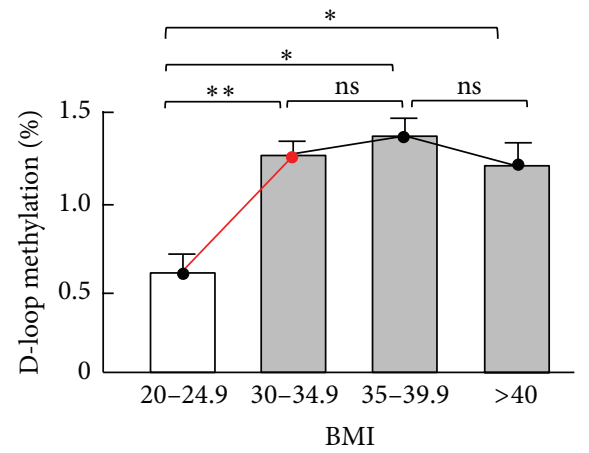

(d)

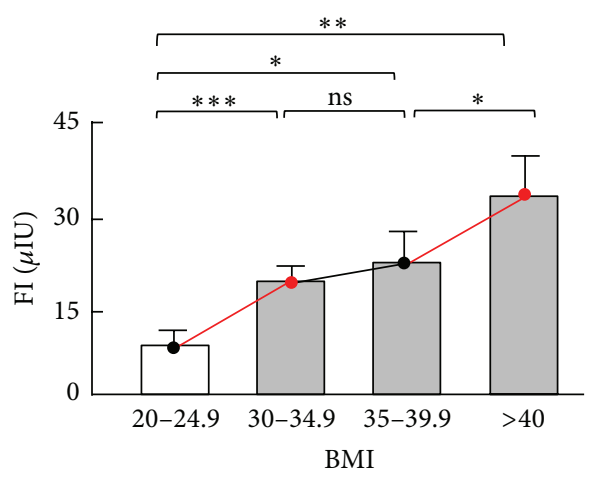

(f)

FIGURE 4: Changes in mitochondrial DNA methylation and insulin sensitivity versus different levels of diabetes risk factor (BMI) or different stages of disease progression. (a) ND6 methylation showed significant correlation with BMI; $n=40$. (b) D-loop methylation showed significant correlation with BMI; $n=40$. (c) ND6 methylation increased drastically from low-risk $(20<$ BMI $<24.9 ; n=8)$ to high-risk $(30$ $<\mathrm{BMI}<34.9 ; n=15)$ stages, with no further significant changes during advanced progression (BMI $>35 ; n=17)$. (d) D-loop methylation increased drastically from low-risk $(20<\mathrm{BMI}<24.9 ; n=8)$ to high-risk $(30<\mathrm{BMI}<34.9 ; n=15)$ stages, with no further significant changes during advanced progression (BMI $>35)$. (e) HOMA-IR increased drastically from low-risk $(20<\mathrm{BMI}<24.9 ; n=8)$ to high-risk $(30<\mathrm{BMI}$ $<34.9 ; n=15$ ) stages and underwent a second significant elevation during advanced progression (from $35<\mathrm{BMI}<39.9$ to $\mathrm{BMI}>40 ; n=9$ and 8, resp.). (f) As an alternative index of insulin resistance, FI increased drastically from low-risk $(20<\mathrm{BMI}<24.9 ; n=8)$ to high-risk $(30$ $<\mathrm{BMI}<34.9 ; n=15$ ) stages and underwent a second significant elevation during advanced progression (from $35<\mathrm{BMI}<39.9$ to $\mathrm{BMI}>40$; $n=9$ and 8 , resp.). ${ }^{*} p<0.05 ;{ }^{* *} p<0.01 ;{ }^{* * *} p<0.001$; ns: not significant. Red color indicates the dramatic changes in a parameter.

methylation was associated with glycemic and lipid profiles [42], but increasing evidence emerges and indicates no or opposite correlations with blood glucose or lipids [41, 4345]. A 6-month exercise intervention was able to significantly reverse the altered DNA methylation in skeletal muscle from first-degree relatives of T2D patients and in adipose tissues from obese and T2D individuals [46, 47]. Upon measuring DNA methylation of long interspersed nucleotide element 1 (LINE-1) by pyrosequencing and global DNA methylation by the luminometric methylation assay (LUMA), Delgado-Cruzata et al. found that changes in weight, diets, or calorie intake affected global DNA methylation [45]. While the phenotype was recapitulated by an intervention study conducted by Martín-Núñez and colleagues [44], Duggan et al. suggested that 12-month lifestyle changes led to significant weight loss with no effects on global DNA methylation [43]. 
These studies have elegantly unraveled an epigenetic link to metabolic diseases, but the relationship between global DNA methylation and environmental and metabolic markers is more complex than expected and warrants further investigation.

Mitochondria underpin metabolic homeostasis [48-50]. We and others have shown that mitochondria undergo genetic, epigenetic, and functional changes in subjects with obesity, insulin resistance, or T2D [5, 18, 50-52]. In the present study, we investigated the relationships between mitochondrial DNA methylation and stage-dependent metabolic markers and diabetes risk index (BMI). It is known that the progression of prediabetes to T2D shows stagedependent features: (1) HOMA insulin sensitivity decreases steeply 5 years prior to T2D occurrence; (2) FG undergoes abrupt increase 2-3 years before T2D onset; (3) significant elevation of Alc is not observed until 1 year before the diagnosis of T2D (Supplemental Figure 1) [24-26]. The different trajectories rank HOMA insulin sensitivity (or insulin resistance, assessed by HOMA-IR) higher than FG and Alc as a potential marker for identifying earlier stage of prediabetes. Indeed, HOMA-IR and FI showed significant correlation with the risk index BMI (Figure 2). Moreover, insulin resistance was significantly associated with the changes in mitochondrial ND6 and D-loop DNA methylation, while the latter showed no correlation with A1c or FG (Figure 3). Based on quartiles of $\mathrm{BMI}$, mitochondrial epigenetic traits underwent significant changes from healthy $(20<\mathrm{BMI}<24.9$, Q1) to obese $(30<$ $\mathrm{BMI}<34.9, \mathrm{Q} 2)$ status, which corresponds to the development of insulin resistance (Figure 4, Supplemental Figure 1). However, no significant change was observed in FG or Alc during the Q1 $\rightarrow$ Q2 transition (Supplemental Figure 5). These findings suggest that mitochondrial epigenetic changes represent an early-stage event in prediabetes progression and is associated with the development of insulin resistance.

The Prevent Diabetes STAT campaign calls for early identification and intervention to prevent the future development of T2D [53]. Thus, our ability to consistently identify prediabetic individuals as early as possible in the disease progression stages will be of utmost importance to address these challenges. In this study we examined the diagnosing power of FG and Alc by following the standards recommended by ADA [12]. FG indicated that $27.6 \%$ of individuals were prediabetic, $1.1 \%$ diabetic, and $71.3 \%$ healthy or normal. By Alc, however, $49.4 \%$ of the tested participants were considered prediabetic and $3.5 \%$ diabetic, leading to a diagnostic rate 1.8 -fold and 3.2-fold higher than FG, respectively (Figure 1). In addition, neither FG nor Alc showed significant correlation with BMI, the primary risk index used for screening (Figure 2), which may account for the wide variation in diagnosis thus highlighting the urgent need for additional diagnosis markers [14, 15]. More importantly, mitochondrial epigenetic signature was significantly correlated with BMI and showed robust changes in early stage of prediabetes progression that corresponds to the development of insulin resistance (Figures 3 and 4). These results suggest that mitochondrial epigenetic marker may have greater potential than Alc or FG to achieve early diagnosis of prediabetes. Future studies seeking to determine the value of mitochondrial epigenetic signatures in accurately diagnosing prediabetes and assessing intervention effectiveness will be of critical importance to fulfill the mission of Prevent Diabetes STAT.

Taken together, effective prevention of T2D requires as-early-as-possible diagnosis of prediabetes for lifestyle intervention. We showed that the current diagnosis indices Alc and FG led to significant discordance, underscoring the urgent need of additional markers to achieve reliable diagnosis. Importantly, we found that the mitochondrial epigenetics signature (DNA methylation in ND6 and D-loop) characteristically signified the early stage of prediabetes when insulin resistance develops. By contrast, advanced stages of prediabetes progression lead to no significant changes in mitochondrial epigenetic signature. Thus, mitochondrial epigenetic changes represent an early-stage event in prediabetes progression, and further studies to systematically examining mitochondrial epigenetic signature in prediabetes diagnosis, particularly in larger-scale cohorts, will be of critical importance.

\section{Abbreviations}

$\begin{array}{ll}\text { Alc: } & \text { Glycated hemoglobin Alc } \\ \text { BMI: } & \text { Body mass index } \\ \text { D-loop: } & \text { Displacement loop } \\ \text { FG: } & \text { Fasting glucose } \\ \text { FI: } & \text { Fasting insulin } \\ \text { HOMA: } & \text { Homeostatic model assessment } \\ \text { HOMA-IR: } & \text { HOMA insulin resistance index } \\ \text { IFG: } & \text { Impaired fasting glucose } \\ \text { LINE-1: } & \text { Long interspersed nucleotide element 1 } \\ \text { LUMA: } & \text { Luminometric methylation assay } \\ \text { ND6: } & \text { Mitochondrial NADH dehydrogenase } 6 \\ \text { T2D: } & \text { Type 2 diabetes. }\end{array}$

\section{Competing Interests}

The authors declare no competing interests.

\section{Acknowledgments}

The authors are grateful to Dr. Carlos J. Pirola for his assistance with MCP assay. Funding for this work was provided in part by USDA National Institute of Food and Agriculture Hatch Project 1007334 (Zhiyong Cheng) and NIH Grant 5R18DK091811-02 (Fabio A. Almeida). Publication of this paper was supported by Virginia Tech's Open Access Subvention Fund.

\section{References}

[1] J. Tuomilehto, J. Lindström, J. G. Eriksson et al., "Prevention of type 2 diabetes mellitus by changes in lifestyle among subjects with impaired glucose tolerance," The New England Journal of Medicine, vol. 344, no. 18, pp. 1343-1350, 2001.

[2] E. S. Schellenberg, D. M. Dryden, B. Vandermeer, C. Ha, and C. Korownyk, "Lifestyle interventions for patients with and at risk for type 2 diabetes: a systematic review and meta-analysis," Annals of Internal Medicine, vol. 159, no. 8, pp. 543-551, 2013. 
[3] P. Tuso, "Prediabetes and lifestyle modification: time to prevent a preventable disease," The Permanente Journal, vol. 18, no. 3, pp. 88-93, 2014.

[4] T. Temelkova-Kurktschiev and T. Stefanov, "Lifestyle and genetics in obesity and type 2 diabetes," Experimental and Clinical Endocrinology and Diabetes, vol. 120, no. 1, pp. 1-6, 2012.

[5] Z. Cheng and F. A. Almeida, "Mitochondrial alteration in type 2 diabetes and obesity: an epigenetic link," Cell Cycle, vol. 13, no. 6, pp. 890-897, 2014.

[6] Z. Cheng, E. M. Schmelz, D. Liu, and M. W. Hulver, "Targeting mitochondrial alterations to prevent type 2 diabetes-evidence from studies of dietary redox-active compounds," Molecular Nutrition and Food Research, vol. 58, no. 8, pp. 1739-1749, 2014.

[7] R. Rabøl, R. Boushel, and F. Dela, "Mitochondrial oxidative function and type 2 diabetes," Applied Physiology, Nutrition and Metabolism, vol. 31, no. 6, pp. 675-683, 2006.

[8] M.-E. Patti and S. Corvera, "The role of mitochondria in the pathogenesis of type 2 diabetes," Endocrine Reviews, vol. 31, no. 3, pp. 364-395, 2010.

[9] J. B. Buse, S. Caprio, W. T. Cefalu et al., "How do we define cure of diabetes?" Diabetes Care, vol. 32, no. 11, pp. 2133-2135, 2009.

[10] C. D. Saudek, "Can diabetes be cured? Potential biological and mechanical approaches," The Journal of the American Medical Association, vol. 301, no. 15, pp. 1588-1590, 2009.

[11] K. M. V. Narayan, J. P. Boyle, T. J. Thompson, E. W. Gregg, and D. F. Williamson, "Effect of BMI on lifetime risk for diabetes in the US," Diabetes Care, vol. 30, no. 6, pp. 1562-1566, 2007.

[12] American Diabetes Association, "Classification and diagnosis of diabetes," Diabetes Care, vol. 38, supplement, pp. S8-S16, 2015.

[13] N. R. Pinelli, A. S. Jantz, E. T. Martin, and L. A. Jaber, "Sensitivity and specificity of glycated hemoglobin as a diagnostic test for diabetes and prediabetes in Arabs," Journal of Clinical Endocrinology and Metabolism, vol. 96, no. 10, pp. E1680-E1683, 2011.

[14] I. B. Hirsch and M. Brownlee, "Beyond hemoglobin Alc-need for additional markers of risk for diabetic microvascular complications," The Journal of the American Medical Association, vol. 303, no. 22, pp. 2291-2292, 2010.

[15] R. M. Cohen and C. J. Lindsell, "When the blood glucose and the $\mathrm{HbA}_{1 \mathrm{c}}$ don't match: turning uncertainty into opportunity," Diabetes Care, vol. 35, no. 12, pp. 2421-2423, 2012.

[16] A. E. Kitabchi, M. Temprosa, W. C. Knowler et al., "Role of insulin secretion and sensitivity in the evolution of type 2 diabetes in the diabetes prevention program effects of lifestyle intervention and metformin," Diabetes, vol. 54, no. 8, pp. 24042414, 2005.

[17] C. Ling and T. Rönn, "Epigenetic adaptation to regular exercise in humans," Drug Discovery Today, vol. 19, no. 7, pp. 1015-1018, 2014.

[18] L. D. Zheng, L. E. Linarelli, L. Liu et al., "Insulin resistance is associated with epigenetic and genetic regulation of mitochondrial DNA in obese humans," Clinical Epigenetics, vol. 7, article 60, 2015.

[19] E. V. Menshikova, V. B. Ritov, L. Fairfull, R. E. Ferrell, D. E. Kelley, and B. H. Goodpaster, "Effects of exercise on mitochondrial content and function in aging human skeletal muscle," Journals of Gerontology-Series A: Biological Sciences and Medical Sciences, vol. 61, no. 6, pp. 534-540, 2006.

[20] N. T. Broskey, A. Boss, E. Fares et al., "Exercise efficiency relates with mitochondrial content and function in older adults," Physiological Reports, vol. 3, no. 6, Article ID e12418, 2015.
[21] N. T. Broskey, C. Greggio, A. Boss et al., "Skeletal muscle mitochondria in the elderly: effects of physical fitness and exercise training," Journal of Clinical Endocrinology and Metabolism, vol. 99, no. 7, pp. 1852-1861, 2014.

[22] B. A. Irving, I. R. Lanza, G. C. Henderson, R. R. Rao, B. M. Spiegelman, and K. Sreekumaran Nair, "Combined training enhances skeletal muscle mitochondrial oxidative capacity independent of age," Journal of Clinical Endocrinology and Metabolism, vol. 100, no. 4, pp. 1654-1663, 2015.

[23] A. W. Gao, C. Cantó, and R. H. Houtkooper, "Mitochondrial response to nutrient availability and its role in metabolic disease," EMBO Molecular Medicine, vol. 6, no. 5, pp. 580-589, 2014.

[24] A. G. Tabák, C. Herder, W. Rathmann, E. J. Brunner, and M. Kivimäki, "Prediabetes: a high-risk state for diabetes development," The Lancet, vol. 379, no. 9833, pp. 2279-2290, 2012.

[25] A. G. Tabák, M. Jokela, T. N. Akbaraly, E. J. Brunner, M. Kivimäki, and D. R. Witte, "Trajectories of glycaemia, insulin sensitivity, and insulin secretion before diagnosis of type 2 diabetes: an analysis from the Whitehall II study," The Lancet, vol. 373, no. 9682, pp. 2215-2221, 2009.

[26] Y. Heianza, Y. Arase, K. Fujihara et al., "Longitudinal trajectories of $\mathrm{HbA}_{1 \mathrm{c}}$ and fasting plasma glucose levels during the development of type 2 diabetes: the Toranomon Hospital Health Management Center Study 7 (TOPICS 7)," Diabetes Care, vol. 35, no. 5, pp. 1050-1052, 2012.

[27] F. A. Almeida, K. A. Pardo, R. W. Seidel et al., "Design and methods of 'diaBEAT-it!': a hybrid preference/randomized control trial design using the RE-AIM framework," Contemporary Clinical Trials, vol. 38, no. 2, pp. 383-396, 2014.

[28] F. X. Xu, X. Zhou, F. Shen, R. Pang, and S. M. Liu, "Decreased peripheral blood mitochondrial DNA content is related to $\mathrm{HbAlc}$, fasting plasma glucose level and age of onset in type 2 diabetes mellitus," Diabetic Medicine, vol. 29, no. 7, pp. e47-e54, 2012.

[29] T. M. Wallace, J. C. Levy, and D. R. Matthews, "Use and abuse of HOMA modeling," Diabetes Care, vol. 27, no. 6, pp. 1487-1495, 2004.

[30] F. F. Zhang, R. M. Santella, M. Wolff, M. A. Kappil, S. B. Markowitz, and A. Morabia, "White blood cell global methylation and IL-6 promoter methylation in association with diet and lifestyle risk factors in a cancer-free population," Epigenetics, vol. 7, no. 6, pp. 606-614, 2012.

[31] K. B. Michels, H. R. Harris, and L. Barault, "Birthweight, maternal weight trajectories and global DNA methylation of LINE-1 repetitive elements," PLoS ONE, vol. 6, no. 9, Article ID e25254, 2011.

[32] C. J. Pirola, T. F. Gianotti, A. L. Burgueño et al., "Epigenetic modification of liver mitochondrial DNA is associated with histological severity of nonalcoholic fatty liver disease," Gut, vol. 62, no. 9, pp. 1356-1363, 2013.

[33] S. Sookoian, M. S. Rosselli, C. Gemma et al., "Epigenetic regulation of insulin resistance in nonalcoholic fatty liver disease: impact of liver methylation of the peroxisome proliferatoractivated receptor $\gamma$ coactivator $1 \alpha$ promoter," Hepatology, vol. 52, no. 6, pp. 1992-2000, 2010.

[34] L.-C. Li and R. Dahiya, "MethPrimer: designing primers for methylation PCRs," Bioinformatics, vol. 18, no. 11, pp. 1427-1431, 2002.

[35] American Diabetes Association, "Consensus development conference on insulin resistance: 5-6 November 1997," Diabetes Care, vol. 21, no. 2, pp. 310-314, 1998. 
[36] V. Pisprasert, K. H. Ingram, M. F. Lopez-Davila, A. J. Munoz, and W. T. Garvey, "Limitations in the use of indices using glucose and insulin levels to predict insulin sensitivity: impact of race and gender and superiority of the indices derived from oral glucose tolerance test in African Americans," Diabetes Care, vol. 36, no. 4, pp. 845-853, 2013.

[37] M. L. Ganz, N. Wintfeld, Q. Li, V. Alas, J. Langer, and M. Hammer, "The association of body mass index with the risk of type 2 diabetes: a case-control study nested in an electronic health records system in the United States," Diabetology \& Metabolic Syndrome, vol. 6, article 50, 2014.

[38] A. Schienkiewitz, M. B. Schulze, K. Hoffmann, A. Kroke, and H. Boeing, "Body mass index history and risk of type 2 diabetes: results from the European Prospective Investigation into Cancer and Nutrition (EPIC)-Potsdam Study," American Journal of Clinical Nutrition, vol. 84, no. 2, pp. 427-433, 2006.

[39] E. W. Gregg, H. Chen, L. E. Wagenknecht et al., "Association of an intensive lifestyle intervention with remission of type 2 diabetes," The Journal of the American Medical Association, vol. 308, no. 23, pp. 2489-2496, 2012.

[40] C. M. Ulrich, A. T. Toriola, L. M. Koepl et al., "Metabolic, hormonal and immunological associations with global DNA methylation among postmenopausal women," Epigenetics, vol. 7, no. 9, pp. 1020-1028, 2012.

[41] V. Turcot, A. Tchernof, Y. Deshaies et al., "LINE-1 methylation in visceral adipose tissue of severely obese individuals is associated with metabolic syndrome status and related phenotypes," Clinical Epigenetics, vol. 4, no. 1, article 10, 2012.

[42] M. S. Pearce, J. C. McConnell, C. Potter et al., "Global LINE-1 DNA methylation is associated with blood glycaemic and lipid profiles," International Journal of Epidemiology, vol. 41, no. 1, pp. 210-217, 2012.

[43] C. Duggan, L. Xiao, M. B. Terry, and A. McTiernan, "No effect of weight loss on LINE-1 methylation levels in peripheral blood leukocytes from postmenopausal overweight women," Obesity, vol. 22, no. 9, pp. 2091-2096, 2014.

[44] G. M. Martín-Núñez, R. Cabrera-Mulero, E. Rubio-Martín et al., "Methylation levels of the SCD1 gene promoter and LINE-1 repeat region are associated with weight change: an intervention study," Molecular Nutrition and Food Research, vol. 58, no. 7, pp. 1528-1536, 2014.

[45] L. Delgado-Cruzata, W. Zhang, J. A. McDonald et al., "Dietary modifications, weight loss, and changes in metabolic markers affect global DNA methylation in Hispanic, African American, and Afro-Caribbean breast cancer survivors," Journal of Nutrition, vol. 145, no. 4, pp. 783-790, 2015.

[46] T. Rönn, P. Volkov, C. Davegårdh et al., "A six months exercise intervention influences the genome-wide DNA methylation pattern in human adipose tissue," PLoS Genetics, vol. 9, no. 6, Article ID e1003572, 2013.

[47] M. D. Nitert, T. Dayeh, P. Volkov et al., "Impact of an exercise intervention on DNA methylation in skeletal muscle from firstdegree relatives of patients with type 2 diabetes," Diabetes, vol. 61, no. 12, pp. 3322-3332, 2012.

[48] Z. Cheng and M. Ristow, "Mitochondria and metabolic homeostasis," Antioxidants \& Redox Signaling, vol. 19, no. 3, pp. 240242, 2013

[49] J. Nunnari and A. Suomalainen, "Mitochondria: in sickness and in health," Cell, vol. 148, no. 6, pp. 1145-1159, 2012.

[50] Z. Cheng, Y. Tseng, and M. F. White, "Insulin signaling meets mitochondria in metabolism," Trends in Endocrinology and Metabolism, vol. 21, no. 10, pp. 589-598, 2010.
[51] Z. Cheng, S. Guo, K. Copps et al., "Foxol integrates insulin signaling with mitochondrial function in the liver," Nature Medicine, vol. 15, no. 11, pp. 1307-1311, 2009.

[52] A. Sleigh, P. Raymond-Barker, K. Thackray et al., "Mitochondrial dysfunction in patients with primary congenital insulin resistance," Journal of Clinical Investigation, vol. 121, no. 6, pp. 2457-2461, 2011.

[53] AMA and CDC, New AMA, CDC Initiative Aims to "Prevent Diabetes STAT”, 2015, http://www.cdc.gov/media/releases/2015/ p0311-diabetes-stat.html. 


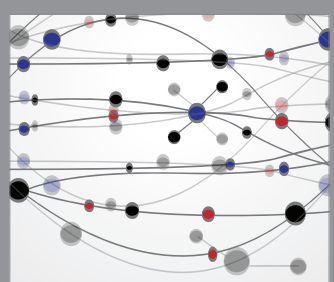

The Scientific World Journal
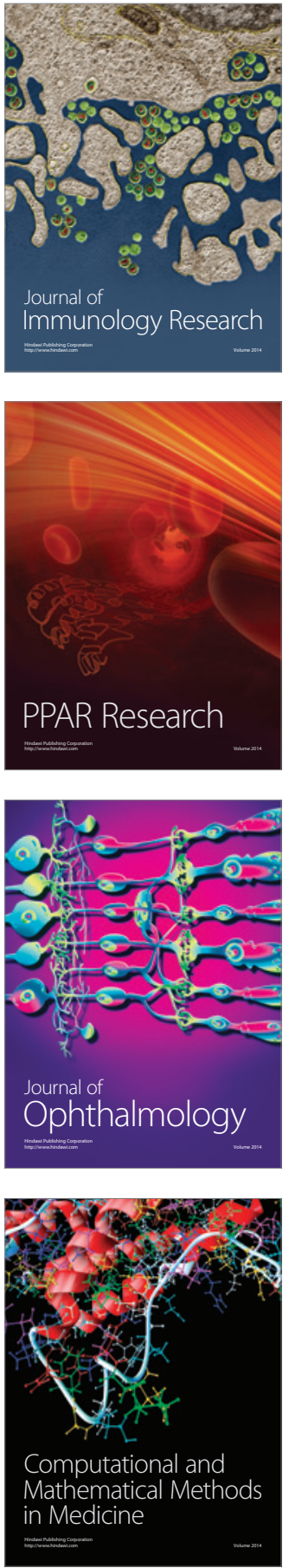

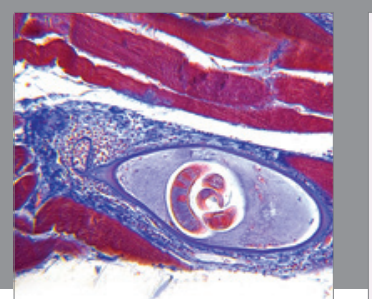

Gastroenterology Research and Practice

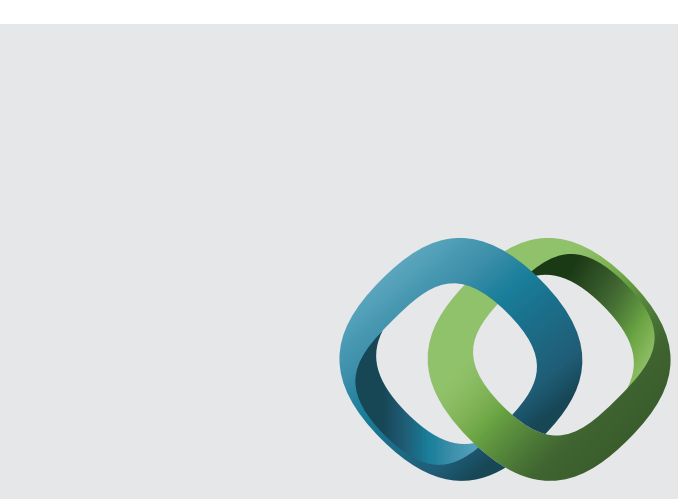

\section{Hindawi}

Submit your manuscripts at

http://www.hindawi.com
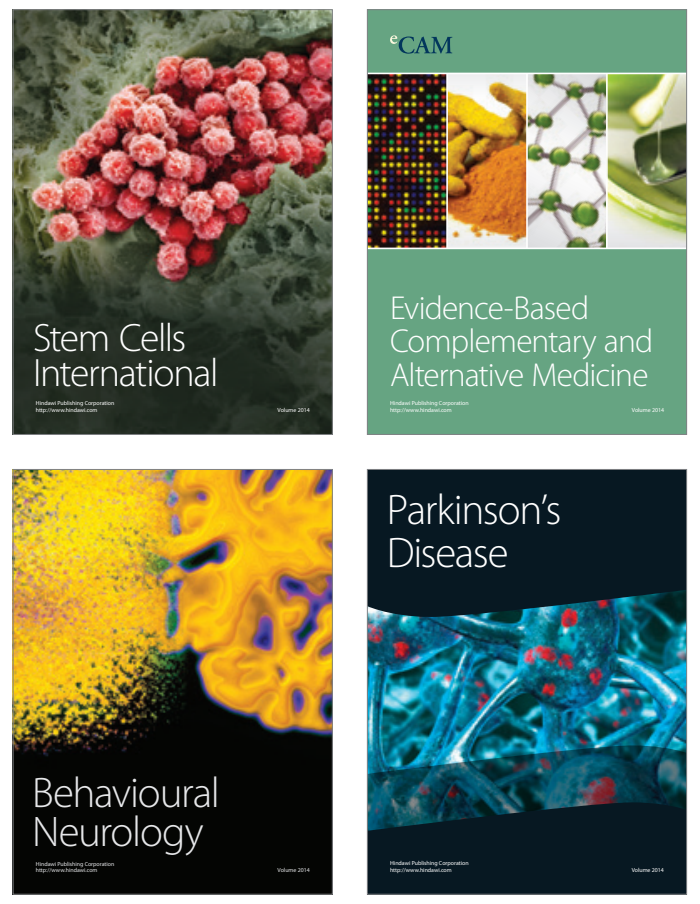
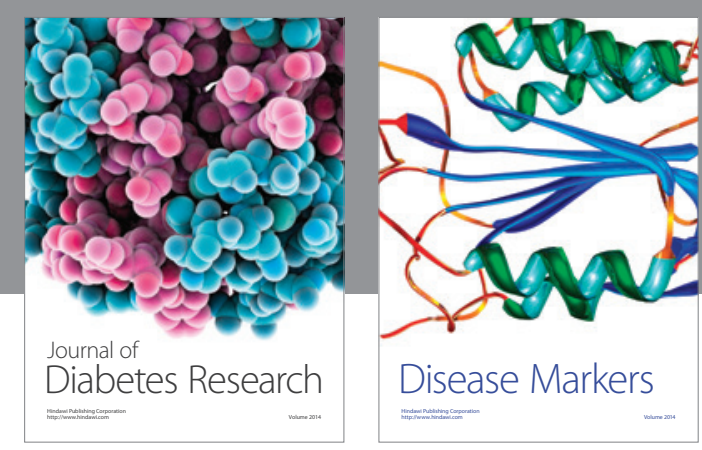

Disease Markers
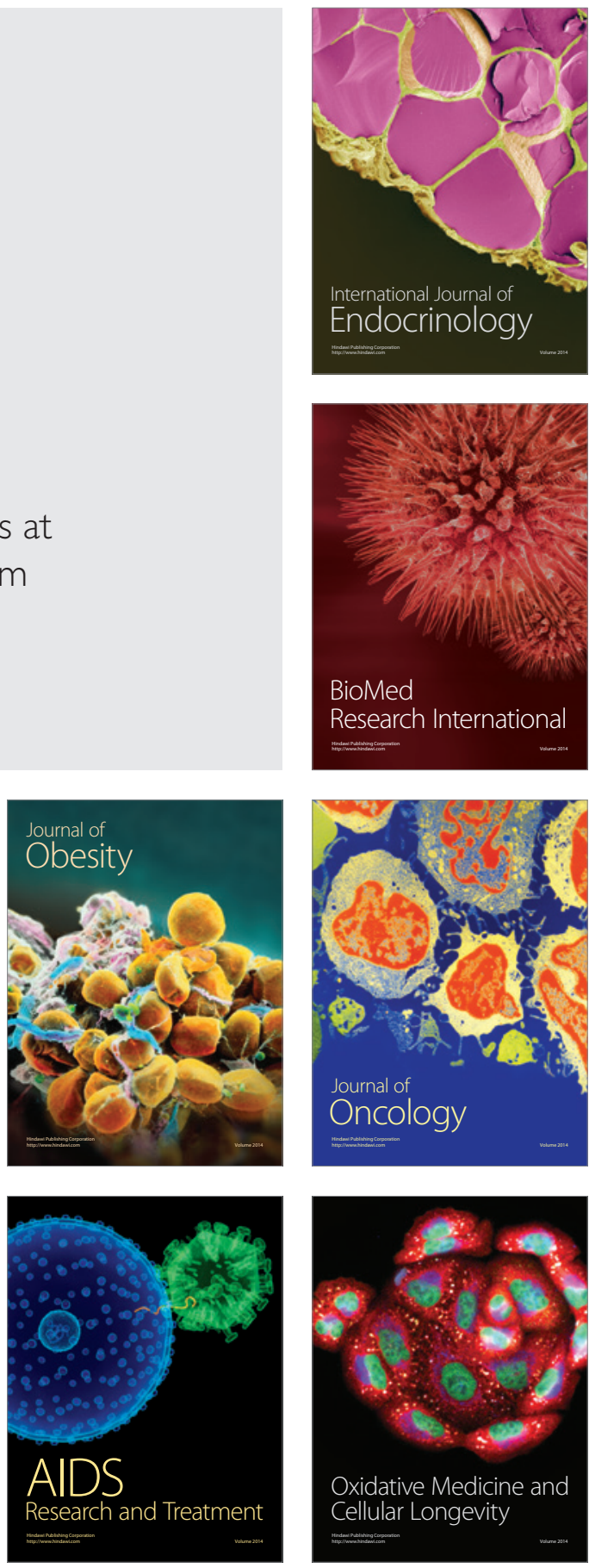